\title{
Pacific
}

Journal of

Mathematics

\section{UNIT INDICES OF SOME IMAGINARY COMPOSITE QUADRATIC FIELDS}

MiKiHITO HIRABAYASHI 


\title{
UNIT INDICES OF SOME IMAGINARY COMPOSITE QUADRATIC FIELDS
}

\author{
Mikihito Hirabayashi
}

Let $K$ be an imaginary abelian number field of type $(2,2,2,2)$ not containing the 8th cyclotomic field. Using the fundamental units of real quadratic subfields of $K$, we give a necessary and sufficient condition for the unit index $Q_{K}$ of $K$ to be equal to 2 .

1. Introduction and results. Let $K$ be an imaginary abelian number field and $K_{0}$ the maximal real subfield of $K$. Let $E$ and $E_{0}$ be the groups of units of $K$ and $K_{0}$, respectively, and let $W$ be the group of roots of unity in $K$. Then we call the group index

$$
Q_{K}=\left[E: W E_{0}\right]
$$

the unit index of $K$.

Using the character group of $K, \mathrm{H}$. Hasse [2] gave sufficient conditions for $Q_{K}$ to be equal to 1 or 2 , by which we can determine $Q_{K}$ for some types of fields $K$. However by his method we cannot always determine $Q_{K}$ for arbitrary $K$, even if $K$ is an imaginary composite quadratic field. (We call a field $K$ a composite quadratic field if $K$ is a composite of quadratic fields.) $\mathrm{K}$. Yoshino and the author $[3,4]$ gave criteria to determine $Q_{K}$ of $K$ with Galois group $\mathrm{Gal}(K / \mathbf{Q})$ of type $(2,2)$ and $(2,2,2)$.

In this paper we extend our previous results $[3,4]$ to the case that $K$ has Galois group $\mathrm{Gal}(K / \mathbf{Q})$ of type $(2,2,2,2)$ and does not contain the 8th cyclotomic field, and then, we give a necessary and sufficient condition for the unit index $Q_{K}$ to be equal to 2 .

Notation. $\mathbf{N}, \mathbf{Z}, \mathbf{Q}$ : the sets of natural numbers, rational integers and rational numbers, respectively,

$=$ : the equality except rational quadratic factors,

$d_{0}, d_{1}, d_{2}, \ldots, d_{7}$ : square-free positive integers such that $d_{4}=$ $d_{2} d_{3}, d_{5}=d_{3} d_{1}, d_{6} \underset{2}{=} d_{1} d_{2}, d_{7} \underset{\overline{2}}{=} d_{1} d_{2} d_{3}$ and $d_{0} \neq d_{i}(i=$ $1,2, \ldots, 7)$,

$K=\mathbf{Q}\left(\sqrt{-d_{0}}, \sqrt{d_{1}}, \sqrt{d_{2}}, \sqrt{d_{3}}\right)$ : an imaginary composite quadratic field of degree 16 , 
$K_{0}=\mathbf{Q}\left(\sqrt{d_{1}}, \sqrt{d_{2}}, \sqrt{d_{3}}\right)$,

$E_{0}^{+}:$the group of totally positive units of $K_{0}$,

$\bar{E}_{0}$ : the group of units $\eta$ of $E_{0}^{+}$such that $K_{0}(\sqrt{\eta})$ is a composite quadratic field,

$$
\begin{array}{ll}
K_{1}=\mathbf{Q}\left(\sqrt{d_{2}}, \sqrt{d_{3}}\right), & K_{2}=\mathbf{Q}\left(\sqrt{d_{3}}, \sqrt{d_{1}}\right), \\
K_{3}=\mathbf{Q}\left(\sqrt{d_{1}}, \sqrt{d_{2}}\right), \quad K_{4}=\mathbf{Q}\left(\sqrt{d_{1}}, \sqrt{d_{2} d_{3}}\right), \\
K_{5}=\mathbf{Q}\left(\sqrt{d_{2}}, \sqrt{d_{3} d_{1}}\right), \quad K_{6}=\mathbf{Q}\left(\sqrt{d_{3}}, \sqrt{d_{1} d_{2}}\right), \\
K_{7}=\mathbf{Q}\left(\sqrt{d_{2} d_{3}}, \sqrt{d_{3} d_{1}}\right), \\
k_{i}=\mathbf{Q}\left(\sqrt{d_{i}}\right)(i=1,2, \ldots, 7), \\
\left\langle\sigma_{i}\right\rangle=\operatorname{Gal}\left(K_{0} / K_{i}\right)(i=1,2, \ldots, 7),
\end{array}
$$

$N(x), \operatorname{Sp}(x)$ : the absolute norm and the absolute trace of $x$, respectively,

$$
A=A\left(e_{1}, e_{2}, e_{3}\right)= \begin{cases}2 d_{1}^{e_{1}} d_{2}^{e_{2}} d_{3}^{e_{3}} & \text { if } d_{0}=1, \\ d_{0} d_{1}^{e_{1}} d_{2}^{e_{2}} d_{3}^{e_{3}} & \text { otherwise }\end{cases}
$$

$\varepsilon_{i}$ : the fundamental unit of $\mathbf{Q}\left(\sqrt{d_{i}}\right), \varepsilon_{i}>1(i=1,2, \ldots, 7)$.

When $N\left(\varepsilon_{i}\right)=+1$, we denote by $\Delta_{i}, \Delta_{i}^{*}$ the square-free parts of $\operatorname{Sp}\left(\varepsilon_{i}+1\right), \operatorname{Sp}\left(\varepsilon_{i}-1\right)$, respectively, and by $m_{i}, n_{i}$ the natural numbers such that $\operatorname{Sp}\left(\varepsilon_{i}+1\right)=\Delta_{i} m_{i}^{2}, \operatorname{Sp}\left(\varepsilon_{i}-1\right)=\Delta_{i}^{*} n_{i}^{2}$. Then we have

$$
\sqrt{\varepsilon_{i}}=\frac{1}{2}\left(m_{i} \sqrt{\Delta_{i}}+n_{i} \sqrt{\Delta_{i}^{*}}\right)
$$

When $d_{i} d_{j}=d_{k}$ with $N\left(\varepsilon_{i}\right)=N\left(\varepsilon_{j}\right)=N\left(\varepsilon_{k}\right)=-1$, we denote by $\Delta_{i j}=\Delta_{j i}$ the square-free integer such that

$$
\Delta_{i j}=\mathrm{Sp}_{\mathbf{Q}\left(\sqrt{d_{t}}, \sqrt{d_{j}}\right) / \mathbf{Q}}\left(\varepsilon_{i} \varepsilon_{j} \varepsilon_{k}-\varepsilon_{i}-\varepsilon_{j}-\varepsilon_{k}\right) .
$$

(We take $(i, j)=(1,2),(1,3),(1,4),(2,3),(2,5),(3,6)$ and $(4,5)$.

When $d_{i} d_{j} d_{k}=d_{l}$ with $N\left(\varepsilon_{i}\right)=N\left(\varepsilon_{j}\right)=N\left(\varepsilon_{k}\right)=N\left(\varepsilon_{l}\right)=-1$ and when $\mathbf{Q}\left(\sqrt{d_{i}}, \sqrt{d_{j}}, \sqrt{d_{k}}\right)=K_{0}$, we denote by $\Delta_{i j k}$ the square-free integer such that

$$
\Delta_{i j k}=\operatorname{Sp}_{K_{0} / \mathrm{Q}}\left(\varepsilon_{i} \varepsilon_{j} \varepsilon_{k} \varepsilon_{l}+1-\sum_{\alpha<\beta} \varepsilon_{\alpha} \varepsilon_{\beta}\right)
$$

where $\alpha, \beta$ run through $i, j, k$ and $l$. 
For a totally positive unit $\eta$ of $K_{0}$ let

(2) $\xi^{*}(\eta)=\eta+\eta^{\sigma_{1}}+2(-1)^{s_{1}} \sqrt{\eta \eta^{\sigma_{1}}}$,

(3) $\theta^{*}(\eta)=\xi^{*}(\eta)+\xi^{*}(\eta)^{\sigma_{2}}+2(-1)^{s_{2}} \sqrt{\xi^{*}(\eta) \xi^{*}(\eta)^{\sigma_{2}}}$,

(4) $d^{*}(\eta)=\theta^{*}(\eta)+\theta^{*}(\eta)^{\sigma_{3}}+2(-1)^{s_{3}} \sqrt{\theta^{*}(\eta) \theta^{*}(\eta)^{\sigma_{3}}} \quad\left(s_{i}=0\right.$ or 1$)$

under the condition that

(5) $\sqrt{\eta \eta^{\sigma_{1}}} \in K_{1}, \quad \sqrt{\xi^{*}(\eta) \xi^{*}(\eta)^{\sigma_{2}}} \in k_{3}$ and $\sqrt{\theta^{*}(\eta) \theta^{*}(\eta)^{\sigma_{3}}} \in \mathbf{Q}$.

We remark that for a totally positive unit $\eta$ of $K_{0}$ this condition (5) is satisfied if and only if $\eta$ is contained in $\bar{E}_{0}$. This remark can be proved by Lemmas 4 and 5 (cf. proof of Theorem 4).

Throughout this paper we assume that $K$ does not contain the 8th cyclotomic field $\mathbf{Q}(\sqrt{-1}, \sqrt{2})$. Our result is the following

MAIN THEOREM. Under the above notation and assumption we have that $Q_{K}=2$ if and only if

$$
\prod_{i} \Delta_{i}^{a_{i}} \cdot \prod_{i, j} \Delta_{i j}^{b_{i j}} \cdot \prod_{i, j, k} \Delta_{i j k}^{c_{i j k}} \cdot d^{*}\left(\eta_{0}\right)^{f}=A\left(e_{1}, e_{2}, e_{3}\right)
$$

for some $a_{i}, b_{i j}, c_{i j k}, f, e_{i}=0,1$ and $\eta_{0} \in \bar{E}_{0}$ represented in the form

$$
\eta_{0}=\sqrt{\prod_{N\left(\varepsilon_{i}\right)=+1} \varepsilon_{i}^{u_{i}}} \cdot \prod_{N\left(\varepsilon_{i}\right)=-1} \varepsilon_{i}^{v_{i}},
$$

where $u_{i}, v_{i}=0$ or 1 . The number of $i$ 's for which $u_{i}=1$ is neither 1 nor 2.

More precisely we have the following Theorems 1-6.

THEOREM 1. In the case that $N\left(\varepsilon_{1}\right)=N\left(\varepsilon_{2}\right)=\cdots=N\left(\varepsilon_{7}\right)=-1$, we have

$$
Q_{K}=2 \Leftrightarrow \Delta_{12}^{b_{1}} \Delta_{23}^{b_{2}} \Delta_{31}^{b_{3}} \Delta_{123}^{c}=A\left(e_{1}, e_{2}, e_{3}\right)
$$

for some $b_{i}, c, e_{i}=0,1$. Especially, if $\sqrt{\Delta_{i j}}$ is contained in $\mathbf{Q}\left(\sqrt{d_{i}}, \sqrt{d_{j}}\right)$ for every $(i, j)$, then $Q_{K}=1$.

THEOREM 2. In the case that $N\left(\varepsilon_{1}\right)=N\left(\varepsilon_{2}\right)=\cdots=N\left(\varepsilon_{6}\right)=-1$ and $N\left(\varepsilon_{7}\right)=+1$, we have

$$
Q_{K}=2 \Leftrightarrow \Delta_{7}^{a} \Delta_{12}^{b_{1}} \Delta_{23}^{b_{2}} \Delta_{31}^{b_{3}}=A\left(e_{1}, e_{2}, e_{3}\right)
$$

for some $a, b_{i}, e_{i}=0,1$. 
THEOREM 3. In the case that $N\left(\varepsilon_{1}\right)=N\left(\varepsilon_{2}\right)=\cdots=N\left(\varepsilon_{5}\right)=-1$ and $N\left(\varepsilon_{6}\right)=N\left(\varepsilon_{7}\right)=+1$, we have

$$
Q_{K}=2 \Leftrightarrow \Delta_{6}^{a_{6}} \Delta_{7}^{a_{7}} \Delta_{23}^{b_{2}} \Delta_{31}^{b_{3}}=A\left(e_{1}, e_{2}, e_{3}\right)
$$

for some $a_{i}, b_{i}, e_{i}=0,1$.

THEOREM 4. (1) In the case that $N\left(\varepsilon_{1}\right)=\cdots=N\left(\varepsilon_{4}\right)=-1$ and $N\left(\varepsilon_{5}\right)=N\left(\varepsilon_{6}\right)=N\left(\varepsilon_{7}\right)=+1$, we have

$$
Q_{K}=2 \Leftrightarrow \Delta_{5}^{a_{5}} \Delta_{6}^{a_{6}} \Delta_{7}^{a_{7}} \Delta_{23}^{b} d^{*}\left(\eta_{0}\right)^{f}=A\left(e_{1}, e_{2}, e_{3}\right)
$$

for some $a_{i}, b, f, e_{i}=0,1$ and $\eta_{0} \in \bar{E}_{0}$ such that

$$
\eta_{0}=\sqrt{\varepsilon_{5} \varepsilon_{6} \varepsilon_{7}} \prod_{i=1}^{4} \varepsilon_{i}^{v_{i}} \quad\left(v_{i}=0 \text { or } 1\right) .
$$

(2) In the case that $N\left(\varepsilon_{1}\right)=N\left(\varepsilon_{2}\right)=N\left(\varepsilon_{3}\right)=N\left(\varepsilon_{7}\right)=-1$ and $N\left(\varepsilon_{4}\right)=N\left(\varepsilon_{5}\right)=N\left(\varepsilon_{6}\right)=+1$, we have

$$
Q_{K}=2 \Leftrightarrow \Delta_{4}^{a_{4}} \Delta_{5}^{a_{5}} \Delta_{6}^{a_{6}} \Delta_{123}^{c} \underset{2}{=} A\left(e_{1}, e_{2}, e_{3}\right)
$$

for some $a_{i}, c, e_{i}=0,1$.

THEOREM 5. (1) In the case that $N\left(\varepsilon_{1}\right)=N\left(\varepsilon_{2}\right)=N\left(\varepsilon_{3}\right)=-1$ and $N\left(\varepsilon_{4}\right)=N\left(\varepsilon_{5}\right)=N\left(\varepsilon_{6}\right)=N\left(\varepsilon_{7}\right)=+1$, we have

$$
Q_{K}=2 \Leftrightarrow \prod_{i=4}^{7} \Delta_{i}^{a_{i}} \cdot d^{*}\left(\eta_{0}\right)^{f}=A\left(e_{1}, e_{2}, e_{3}\right)
$$

for some $a_{i}, f, e_{i}=0,1$ and $\eta_{0} \in \bar{E}_{0}$ such that

$$
\frac{\eta_{0}}{\prod_{i=1}^{3} \varepsilon_{i}^{v_{i}}}=\sqrt{\varepsilon_{4} \varepsilon_{5} \varepsilon_{7}}, \sqrt{\varepsilon_{5} \varepsilon_{6} \varepsilon_{7}} \text { or } \sqrt{\varepsilon_{6} \varepsilon_{4} \varepsilon_{7}} \quad\left(v_{i}=0 \text { or } 1\right) .
$$

(2) In the case that $N\left(\varepsilon_{1}\right)=N\left(\varepsilon_{2}\right)=N\left(\varepsilon_{6}\right)=-1$ and the others $N\left(\varepsilon_{i}\right)=+1$, we have

$$
Q_{K}=2 \Leftrightarrow \prod_{N\left(\varepsilon_{i}\right)=+1} \Delta_{i}^{a_{i}} \cdot \Delta_{12}^{b} \cdot d^{*}\left(\eta_{0}\right)^{f}=A\left(e_{1}, e_{2}, e_{3}\right)
$$

for some $a_{i}, b, f, e_{i}=0,1$ and $\eta_{0} \in \bar{E}_{0}$ such that

$$
\begin{aligned}
& \frac{\eta_{0}}{\prod_{N\left(\varepsilon_{i}\right)=-1} \varepsilon_{i}^{v_{i}}}= \sqrt{\varepsilon_{3} \varepsilon_{4} \varepsilon_{5} \varepsilon_{7}}, \sqrt{\varepsilon_{3} \varepsilon_{4} \varepsilon_{5}}, \sqrt{\varepsilon_{3} \varepsilon_{4} \varepsilon_{7}}, \\
& \sqrt{\varepsilon_{3} \varepsilon_{5} \varepsilon_{7}} \text { or } \sqrt{\varepsilon_{4} \varepsilon_{5} \varepsilon_{7}} \quad\left(v_{i}=0 \text { or } 1\right) .
\end{aligned}
$$


THEOREM 6. In the case that $N\left(\varepsilon_{3}\right)=N\left(\varepsilon_{4}\right)=\cdots=N\left(\varepsilon_{7}\right)=+1$, we have

$$
Q_{K}=2 \Leftrightarrow \prod_{N\left(\varepsilon_{l}\right)=+1} \Delta_{i}^{a_{t}} \cdot d^{*}\left(\eta_{0}\right)^{f}=A\left(e_{1}, e_{2}, e_{3}\right)
$$

for some $a_{i}, f, e_{i}=0,1$ and $\eta_{0} \in \bar{E}_{0}$ such that

$$
\frac{\eta_{0}}{\sqrt{\prod_{N\left(\varepsilon_{l}\right)=+1} \varepsilon_{i}^{u_{i}}}}=\varepsilon_{1}^{v_{1}} \varepsilon_{2}^{v_{2}}, \varepsilon_{1}^{v_{1}} \text { or } 1 \quad\left(u_{i}, v_{i}=0 \text { or } 1\right)
$$

according as $N\left(\varepsilon_{1}\right)=N\left(\varepsilon_{2}\right)=-1 ; N\left(\varepsilon_{1}\right)=-1$ and $N\left(\varepsilon_{2}\right)=+1$; or $N\left(\varepsilon_{1}\right)=N\left(\varepsilon_{2}\right)=+1$. The number of $i$ 's for which $u_{i}=1$ is neither 1 nor 2.

REMARK 1. In Main Theorem $\eta_{0}$ is not represented in the form

$$
\eta_{0}=\sqrt{\varepsilon_{i} \varepsilon_{j} \varepsilon_{k}} \cdot \prod_{N\left(\varepsilon_{l}\right)=-1} \varepsilon_{l}^{v_{l}}
$$

where $N\left(\varepsilon_{i}\right)=N\left(\varepsilon_{j}\right)=N\left(\varepsilon_{k}\right)=+1$ and $d_{i} d_{j}=d_{k}$ (cf. proof of Case (2) of Theorem 4).

REMARK 2. For some $\eta_{0} \in \bar{E}_{0}$ we can actually calculate the rational integers $d^{*}\left(\eta_{0}\right)$ defined by (4). For example, we can obtain the following: Suppose that $N\left(\varepsilon_{1}\right)=N\left(\varepsilon_{2}\right)=N\left(\varepsilon_{3}\right)=+1$ and that $\eta_{0}=\sqrt{\varepsilon_{1} \varepsilon_{2} \varepsilon_{3}}$ is totally positive. Then $\eta_{0} \in \bar{E}_{0}$ if and only if

$$
\Delta_{1}=d_{2} d_{3}, \quad \Delta_{2}=d_{3} d_{1}, \quad \Delta_{3}=d_{1} d_{2} .
$$

If this condition (6) is satisfied, we have

$$
\begin{aligned}
d^{*}\left(\eta_{0}\right)= & m_{1} m_{2} m_{3} \sqrt{\Delta_{1} \Delta_{2} \Delta_{3}} \\
& +2 \Delta_{1}^{*}\left\{(-1)^{s_{1}} n_{2} n_{3}+(-1)^{s_{2}} n_{3} n_{1}+(-1)^{s_{3}} n_{1} n_{2}\right\} \\
& -8(-1)^{s_{1}+s_{2}+s_{3}} \quad\left(s_{i}=0 \text { or } 1\right)
\end{aligned}
$$

where $\Delta_{i}, \Delta_{i}^{*}, m_{i}, n_{i}$ and $s_{i}$ are as in the notation.

2. Properties of $\bar{E}_{0}$ and lemmas on $(2,2)$-extensions. In this section we give a proposition and some lemmas which will be used in the proofs of theorems.

Let $\langle x, y, \ldots\rangle$ be a group generated by $x, y, \ldots$. Let $E_{0}^{*}$ be the subgroup of $E_{0}$ generated by the units of $\mathbf{Q}\left(\sqrt{d_{i}}\right)$ for $i=1,2, \ldots, 7$. Let $\left(E_{0}^{*}\right)^{+}$be the subgroup of $E_{0}$ generated by totally positive units of $E_{0}^{*}$, i.e., $\left(E_{0}^{*}\right)^{+}=E_{0}^{*} \cap E_{0}^{+}$. 
Proposition 1. (1) If $N\left(\varepsilon_{1}\right)=\cdots=N\left(\varepsilon_{7}\right)=-1$, then

$$
\left(E_{0}^{*}\right)^{+}=\left\langle\varepsilon_{2} \varepsilon_{3} \varepsilon_{4}, \varepsilon_{3} \varepsilon_{1} \varepsilon_{5}, \varepsilon_{1} \varepsilon_{2} \varepsilon_{6}, \varepsilon_{1} \varepsilon_{2} \varepsilon_{3} \varepsilon_{7}\right\rangle E_{0}^{* 2} .
$$

(2) If $N\left(\varepsilon_{1}\right)=\cdots=N\left(\varepsilon_{6}\right)=-1$ and $N\left(\varepsilon_{7}\right)=+1$, then

$$
\left(E_{0}^{*}\right)^{+}=\left\langle\varepsilon_{2} \varepsilon_{3} \varepsilon_{4}, \varepsilon_{3} \varepsilon_{1} \varepsilon_{5}, \varepsilon_{1} \varepsilon_{2} \varepsilon_{6}, \varepsilon_{7}\right\rangle E_{0}^{* 2} .
$$

(3) If $N\left(\varepsilon_{1}\right)=\cdots=N\left(\varepsilon_{5}\right)=-1$ and $N\left(\varepsilon_{6}\right)=N\left(\varepsilon_{7}\right)=+1$, then

$$
\left(E_{0}^{*}\right)^{+}=\left\langle\varepsilon_{2} \varepsilon_{3} \varepsilon_{4}, \varepsilon_{3} \varepsilon_{1} \varepsilon_{5}, \varepsilon_{6}, \varepsilon_{7}\right\rangle E_{0}^{* 2} .
$$

$\left(4_{1}\right)$ If $N\left(\varepsilon_{1}\right)=\cdots=N\left(\varepsilon_{4}\right)=-1$ and $N\left(\varepsilon_{5}\right)=N\left(\varepsilon_{6}\right)=N\left(\varepsilon_{7}\right)=$ +1 , then

$$
\left(E_{0}^{*}\right)^{+}=\left\langle\varepsilon_{2} \varepsilon_{3} \varepsilon_{4}, \varepsilon_{5}, \varepsilon_{6}, \varepsilon_{7}\right\rangle E_{0}^{* 2} .
$$

$\left(4_{2}\right)$ If $N\left(\varepsilon_{1}\right)=N\left(\varepsilon_{2}\right)=N\left(\varepsilon_{3}\right)=N\left(\varepsilon_{7}\right)=-1$ and $N\left(\varepsilon_{4}\right)=$ $N\left(\varepsilon_{5}\right)=N\left(\varepsilon_{6}\right)=+1$, then

$$
\left(E_{0}^{*}\right)^{+}=\left\langle\varepsilon_{1} \varepsilon_{2} \varepsilon_{3} \varepsilon_{7}, \varepsilon_{4}, \varepsilon_{5}, \varepsilon_{6}\right\rangle E_{0}^{* 2} .
$$

$\left(5_{1}\right)$ If $N\left(\varepsilon_{1}\right)=N\left(\varepsilon_{2}\right)=N\left(\varepsilon_{3}\right)=-1$ and $N\left(\varepsilon_{4}\right)=\cdots=N\left(\varepsilon_{7}\right)=$ +1 , then

$$
\left(E_{0}^{*}\right)^{+}=\left\langle\varepsilon_{4}, \varepsilon_{5}, \varepsilon_{6}, \varepsilon_{7}\right\rangle E_{0}^{* 2} .
$$

$\left(5_{2}\right)$ If $N\left(\varepsilon_{1}\right)=N\left(\varepsilon_{2}\right)=N\left(\varepsilon_{6}\right)=-1$ and the others $N\left(\varepsilon_{i}\right)=+1$, then

$$
\left(E_{0}^{*}\right)^{+}=\left\langle\varepsilon_{1} \varepsilon_{2} \varepsilon_{6}, \varepsilon_{3}, \varepsilon_{4}, \varepsilon_{5}, \varepsilon_{7}\right\rangle E_{0}^{* 2} .
$$

(6) If $N\left(\varepsilon_{1}\right)=N\left(\varepsilon_{2}\right)=-1$ and $N\left(\varepsilon_{3}\right)=\cdots=N\left(\varepsilon_{7}\right)=+1$, then

$$
\left(E_{0}^{*}\right)^{+}=\left\langle\varepsilon_{3}, \varepsilon_{4}, \varepsilon_{5}, \varepsilon_{6}, \varepsilon_{7}\right\rangle E_{0}^{* 2} \text {. }
$$

(7) If $N\left(\varepsilon_{1}\right)=-1$ and $N\left(\varepsilon_{2}\right)=\cdots=N\left(\varepsilon_{7}\right)=+1$, then

$$
\left(E_{0}^{*}\right)^{+}=\left\langle\varepsilon_{2}, \varepsilon_{3}, \ldots, \varepsilon_{7}\right\rangle E_{0}^{* 2} \text {. }
$$

(8) If $N\left(\varepsilon_{1}\right)=\cdots=N\left(\varepsilon_{7}\right)+1$, then

$$
\left(E_{0}^{*}\right)^{+}=\left\langle\varepsilon_{1}, \varepsilon_{2}, \ldots, \varepsilon_{7}\right\rangle E_{0}^{* 2} \text {. }
$$

Proof. We only prove the case (1), because the other cases are proved in the same way.

For an element $\alpha \neq 0$ of $K$ we define $s(\alpha)=0$ or 1 by $(-1)^{s(\alpha)}=$ $\alpha /|\alpha|$.

For $\eta \in\left(E_{0}^{*}\right)^{+}$, putting $\eta=\varepsilon_{1}^{x_{1}} \varepsilon_{2}^{x_{2}} \cdots \varepsilon_{7}^{x_{7}}\left(x_{i} \in \mathbf{Z}\right)$, we have a system of simultaneous linear equations

$$
\left\{\begin{array}{c}
s\left(\varepsilon_{1}\right) x_{1}+s\left(\varepsilon_{2}\right) x_{2}+\cdots+s\left(\varepsilon_{7}\right) x_{7} \equiv 0 \\
s\left(\varepsilon_{1}^{\sigma_{1}}\right) x_{1}+s\left(\varepsilon_{2}^{\sigma_{1}}\right) x_{2}+\cdots+s\left(\varepsilon_{7}^{\sigma_{1}}\right) x_{7} \equiv 0 \\
\cdots \\
s\left(\varepsilon_{1}^{\sigma_{7}}\right) x_{1}+s\left(\varepsilon_{2}^{\sigma_{7}}\right) x_{2}+\cdots+s\left(\varepsilon_{7}^{\sigma_{7}}\right) x_{7} \equiv 0 .
\end{array}\right.
$$


By Gauss-Jordan elimination (see, for example, H. Anton, Elementary Linear Algebra, John Wiley \& Sons (1973), pp. 18-20) we see that this system has the following four linearly independent solutions:

$$
\left(\begin{array}{l}
x_{1} \\
x_{2} \\
x_{3} \\
x_{4} \\
x_{5} \\
x_{6} \\
x_{7}
\end{array}\right)=\left(\begin{array}{l}
0 \\
1 \\
1 \\
1 \\
0 \\
0 \\
0
\end{array}\right),\left(\begin{array}{l}
1 \\
0 \\
1 \\
0 \\
1 \\
0 \\
0
\end{array}\right),\left(\begin{array}{l}
1 \\
1 \\
0 \\
0 \\
0 \\
1 \\
0
\end{array}\right),\left(\begin{array}{l}
1 \\
1 \\
1 \\
0 \\
0 \\
0 \\
1
\end{array}\right) .
$$

To these solutions correspond units $\varepsilon_{2} \varepsilon_{3} \varepsilon_{4}, \varepsilon_{3} \varepsilon_{1} \varepsilon_{5}, \varepsilon_{1} \varepsilon_{2} \varepsilon_{6}, \varepsilon_{1} \varepsilon_{2} \varepsilon_{3} \varepsilon_{7}$ respectively. Thus we have

$$
\left(E_{0}^{*}\right)^{+}=\left\langle\varepsilon_{2} \varepsilon_{3} \varepsilon_{4}, \varepsilon_{3} \varepsilon_{1} \varepsilon_{5}, \varepsilon_{1} \varepsilon_{2} \varepsilon_{6}, \varepsilon_{1} \varepsilon_{2} \varepsilon_{3} \varepsilon_{7}\right\rangle E_{0}^{* 2} .
$$

In general, let $K / k$ be a $(2,2)$-extension with Galois group $\operatorname{Gal}(K / k)=\langle\sigma, \tau\rangle$. Then, as used by H. Wada [6], we have

$$
\alpha^{2}=\frac{\alpha^{1+\sigma} \alpha^{1+\tau}}{\left(\alpha^{\sigma}\right)^{1+\sigma \tau}}
$$

for $\alpha \in K, \alpha \neq 0$. By this simple formula we see that $E_{0}^{4} \subseteq E_{0}^{*}$. Moreover, we have $\bar{E}_{0}^{2} \subseteq E_{0}^{*}$ by the following

LeMmA 1. Let $\eta \in \bar{E}_{0}$ and put $\eta^{4}=\varepsilon_{1}^{x_{1}} \varepsilon_{2}^{x_{2}} \cdots \varepsilon_{7}^{x_{7}}\left(x_{i} \in \mathbf{Z}\right)$. Then, every $x_{i}$ is even.

Proof. Since $K_{0}(\sqrt{\eta})=K_{0}(\sqrt{d})$ for some $d \in \mathbf{N}$, we can put $\eta=$ $d \alpha_{0}^{2}\left(\alpha_{0} \in K_{0}\right)$. Taking the norm $N_{K_{0} / k_{i}}$ of $\varepsilon_{1}^{x_{1}} \varepsilon_{2}^{x_{2}} \cdots \varepsilon_{7}^{x_{7}}=d^{4} \alpha_{0}^{8}$, we have $\varepsilon_{i}^{4 x_{i}}=d^{16} N_{K_{0} / k_{t}}\left(\alpha_{0}\right)^{8}$. This implies that $x_{i}$ is even.

LEMMA 2. Let $\eta \in \bar{E}_{0}$ and put

$$
\eta^{2}=\varepsilon_{1}^{x_{1}} \varepsilon_{2}^{x_{2}} \cdots \varepsilon_{7}^{x_{7}} \quad\left(x_{i} \in \mathbf{Z}\right) .
$$

Then, all $x_{i}$ are even or at least three $x_{i}$ 's are odd.

Proof. For the simplicity we denote by $N_{i}$ the norm $N_{K_{0} / K_{i}}$ for each $i$.

First, for example, we assume that $x_{1} \equiv 1, x_{i} \equiv 0(\bmod 2)(i=$ $2,3, \ldots, 7)$. Taking the norm $N_{3}$ of the equation (7), we have $N_{3}(\eta)=\varepsilon_{1}^{x_{1}} \varepsilon_{2}^{x_{2}} \varepsilon_{6}^{x_{6}} \in K_{3}$. On the other hand, putting $\eta=d \alpha_{0}^{2}(d \in \mathbf{N}$, 
$\left.\alpha_{0} \in K_{0}\right)$, we have $N_{3}(\eta)=d^{2} N_{3}\left(\alpha_{0}\right)^{2}$. Therefore, $\sqrt{\varepsilon_{1}}$ is contained in $K_{3}=\mathbf{Q}\left(\sqrt{d_{1}}, \sqrt{d_{2}}\right)$. In the same way, taking the norm $N_{2}$ of (7), we see that $\sqrt{\varepsilon_{1}}$ is contained in $K_{2}=\mathbf{Q}\left(\sqrt{d_{3}}, \sqrt{d_{1}}\right)$. Thus $\sqrt{\varepsilon_{1}}$ is contained in $K_{2} \cap K_{3}=\mathbf{Q}\left(\sqrt{d_{1}}\right)$, which is impossible.

Secondly, for example, we assume that $x_{1} \equiv x_{2} \equiv 1, x_{i} \equiv 0(\bmod 2)$ $(i=3,4, \ldots, 7)$. Taking the norms $N_{2}, N_{4}$ of (7), we see that $\sqrt{\varepsilon_{1}}$ is contained in $\mathbf{Q}\left(\sqrt{d_{1}}\right)$, which is also impossible.

Thus there is no case that exactly one or two of $x_{i}$ are odd.

LEMMA 3. Let $\eta \in \bar{E}_{0}$ and put

$$
\eta^{2}=\varepsilon_{1}^{x_{1}} \varepsilon_{2}^{x_{2}} \cdots \varepsilon_{7}^{x_{7}} \quad\left(x_{i} \in \mathbf{Z}\right) .
$$

(1) If there exists an even $x_{i}$, then $N\left(\varepsilon_{j}\right)=+1$ for each odd $x_{j}$.

(2) If there exists " $i$ " for which $x_{i} \equiv 0(\bmod 2)$ or $N\left(\varepsilon_{i}\right)=+1$, then $x_{j}$ is even when $N\left(\varepsilon_{j}\right)=-1$.

(3) If $x_{1} \equiv x_{2} \equiv \cdots \equiv x_{7} \equiv 1(\bmod 2)$, then $N\left(\varepsilon_{1}\right)=N\left(\varepsilon_{2}\right)=\cdots=$ $N\left(\varepsilon_{7}\right)$.

Proof. (1) Suppose that $x_{1} \equiv 1, x_{2} \equiv 0(\bmod 2)$. Taking the norm $N_{3}$ of $(8)$, we have $N_{3}(\eta)=\varepsilon_{1}^{x_{1}} \varepsilon_{2}^{x_{2}} \varepsilon_{6}^{x_{6}}$. Again, taking the norms $N_{1}, N_{2}$ of this equation, we have by $\eta \gg 0$ that

$$
\begin{aligned}
& N_{1}\left(N_{3}(\eta)\right)=N\left(\varepsilon_{1}\right)^{x_{1}} \varepsilon_{2}^{2 x_{2}} N\left(\varepsilon_{6}\right)^{x_{6}}>0, \\
& N_{2}\left(N_{3}(\eta)\right)=\varepsilon_{1}^{2 x_{1}} N\left(\varepsilon_{2}\right)^{x_{2}} N\left(\varepsilon_{6}\right)^{x_{6}}>0 .
\end{aligned}
$$

Hence $N\left(\varepsilon_{6}\right)^{x_{6}}=+1$ and then $N\left(\varepsilon_{1}\right)=+1$.

(2) We suppose that $x_{1} \equiv 0(\bmod 2)$ or $N\left(\varepsilon_{1}\right)=+1$ and that $N\left(\varepsilon_{2}\right)=-1$.

Taking the norm $N_{3}$ of (8), we have $N_{3}(\eta)=\varepsilon_{1}^{x_{1}} \varepsilon_{2}^{x_{2}} \varepsilon_{6}^{x_{6}}$. Again, taking the norm $N_{6}$ of this equation, we have

$$
N_{6}\left(N_{3}(\eta)\right)=N\left(\varepsilon_{1}\right)^{x_{1}} N\left(\varepsilon_{2}\right)^{x_{2}} \varepsilon_{6}^{2 x_{6}}>0,
$$

and so $x_{2} \equiv 0(\bmod 2)$.

(3) Taking the norm $N_{1}$ of (8), we have $N_{1}(\eta)=\varepsilon_{2}^{x_{2}} \varepsilon_{3}^{x_{3}} \varepsilon_{4}^{x_{4}}$. Moreover, taking the norms $N_{2}, N_{3}$ of this equation, we have

$$
\begin{aligned}
& N_{2}\left(N_{1}(\eta)\right)=N\left(\varepsilon_{2}\right)^{x_{2}} \varepsilon_{3}^{2 x_{3}} N\left(\varepsilon_{4}\right)^{x_{4}}>0 \\
& N_{3}\left(N_{1}(\eta)\right)=\varepsilon_{2}^{2 x_{2}} N\left(\varepsilon_{3}\right)^{x_{3}} N\left(\varepsilon_{4}\right)^{x_{4}}>0
\end{aligned}
$$

Then $N\left(\varepsilon_{2}\right)=N\left(\varepsilon_{3}\right)=N\left(\varepsilon_{4}\right)$. 
In the same way, taking the norms $N_{2}, N_{3}, N_{6}$ of (8), we obtain $N\left(\varepsilon_{3}\right)=N\left(\varepsilon_{1}\right)=N\left(\varepsilon_{5}\right), N\left(\varepsilon_{1}\right)=N\left(\varepsilon_{2}\right)=N\left(\varepsilon_{6}\right), N\left(\varepsilon_{3}\right)=N\left(\varepsilon_{6}\right)=$ $N\left(\varepsilon_{7}\right)$.

For a field $k$ we denote by " $\overline{2}$ in $k$ " the equality except a square of a number of $k$.

LemMA 4 (F. Halter-Koch [1, Satz 1]). Let $K_{1}$ be a field with $\overline{\operatorname{char}}\left(K_{1}\right) \neq 2$. Let $K_{0}$ be a quadratic extension of $K_{1}$ and $K_{0}\left(\sqrt{\eta_{0}}\right)\left(\eta_{0} \in K_{0}\right)$ a biquadratic (quartic) extension of $K_{1}$. Then $K_{0}\left(\sqrt{\eta_{0}}\right) / K_{1}$ is bicyclic if and only if $N_{K_{0} / K_{1}}\left(\eta_{0}\right)=1$ in $K_{1}$.

By this Lemma 4 we can easily obtain

LEMMA 5. Let $K_{1}$ be an algebraic number field and $K_{0}$ a quadratic extension of $K_{1}$. Let $K_{0}\left(\sqrt{\eta_{0}}\right)\left(\eta_{0} \in K_{0}, \eta_{0} \notin K_{1}\right)$ be a biquadratic bicyclic extension of $K_{1}$ with $\operatorname{Gal}\left(K_{0}\left(\sqrt{\eta_{0}}\right) / K_{1}\right)=\langle\sigma, \tau\rangle$ and $\operatorname{Gal}\left(K_{0}\left(\sqrt{\eta_{0}}\right) / K_{0}\right)=\langle\tau\rangle$. Let $F$ be the intermediate field of $K_{0}\left(\sqrt{\eta_{0}}\right) / K_{1}$ fixed by $\sigma$. Then we have

$$
F=K_{1}\left(\sqrt{\eta_{0}}+{\sqrt{\eta_{0}}}^{\sigma}\right) \text {. }
$$

3. Proof of theorems. For the proof of Main Theorem, it is enough to prove Theorems 1-6, because the cases of Proposition 1 cover all the possible cases of the combination of $N\left(\varepsilon_{i}\right)= \pm 1$.

Let $K^{\prime}$ be the quadratic extension of $K$ generated by a primitive $2^{n+1}$ th root of unity, $2^{n} \| \# W$, and let $K_{0}^{\prime}$ be the maximal real subfield of $K^{\prime}$.

When $d_{i} d_{j}=d_{k}$ and $N\left(\varepsilon_{i}\right)=N\left(\varepsilon_{j}\right)=N\left(\varepsilon_{k}\right)=-1$, let

$$
\eta_{i j}=\varepsilon_{i} \varepsilon_{j} \varepsilon_{k}, \quad \xi_{i j}=\varepsilon_{i} \varepsilon_{j} \varepsilon_{k}-\varepsilon_{i}-\varepsilon_{j}-\varepsilon_{k} .
$$

Then it follows from T. Kubota $[5, \S 5]$ that

$$
\eta_{i j} \operatorname{Sp}\left(\xi_{i j}\right)=\xi_{i j}^{2} \text {. }
$$

For the multi-quadratic field $K_{0}=\mathbf{Q}\left(\sqrt{d_{1}}, \sqrt{d_{2}}, \sqrt{d_{3}}\right)$, we can prove:

LEMMA 6. Suppose that $N\left(\varepsilon_{1}\right)=N\left(\varepsilon_{2}\right)=N\left(\varepsilon_{3}\right)=N\left(\varepsilon_{7}\right)=-1$. Let

$$
\begin{aligned}
& \eta=\eta_{123}=\varepsilon_{1} \varepsilon_{2} \varepsilon_{3} \varepsilon_{7}, \\
& \xi=\xi_{123}=\eta+1-\left(\varepsilon_{1} \varepsilon_{2}+\varepsilon_{2} \varepsilon_{3}+\varepsilon_{3} \varepsilon_{1}+\varepsilon_{1} \varepsilon_{7}+\varepsilon_{2} \varepsilon_{7}+\varepsilon_{3} \varepsilon_{7}\right) .
\end{aligned}
$$


Then we have

$$
\eta \mathrm{Sp}(\xi)=\xi^{2}
$$

Proof. Since

$$
\xi^{\sigma_{1}}=\varepsilon_{1}^{\prime} \varepsilon_{2} \varepsilon_{3} \varepsilon_{7}^{\prime}+1-\varepsilon_{1}^{\prime} \varepsilon_{2}-\varepsilon_{2} \varepsilon_{3}-\varepsilon_{3} \varepsilon_{1}^{\prime}-\varepsilon_{1}^{\prime} \varepsilon_{7}^{\prime}-\varepsilon_{2} \varepsilon_{7}^{\prime}-\varepsilon_{3} \varepsilon_{7}^{\prime},
$$

it holds that $\varepsilon_{1} \varepsilon_{7} \xi^{\sigma_{1}}=-\xi$, where $\varepsilon^{\prime}$ is the conjugate of $\varepsilon$ with respect to $\mathbf{Q}$. In the same way we have

$$
\begin{gathered}
\varepsilon_{2} \varepsilon_{7} \xi^{\sigma_{2}}=\varepsilon_{3} \varepsilon_{7} \xi^{\sigma_{3}}=\varepsilon_{2} \varepsilon_{3} \xi^{\sigma_{4}}=\varepsilon_{3} \varepsilon_{1} \xi^{\sigma_{5}}=\varepsilon_{1} \varepsilon_{2} \xi^{\sigma_{6}}=-\xi, \\
\varepsilon_{1} \varepsilon_{2} \varepsilon_{3} \varepsilon_{7} \xi^{\sigma_{7}}=\xi .
\end{gathered}
$$

Therefore

$$
\begin{aligned}
\mathrm{Sp}_{K_{0} / \mathbf{Q}}(\xi) & =\xi+\xi^{\sigma_{1}}+\cdots+\xi^{\sigma_{7}} \\
& =\xi\left(1-\sum_{i<j} \frac{1}{\varepsilon_{i} \varepsilon_{j}}+\frac{1}{\varepsilon_{1} \varepsilon_{2} \varepsilon_{3} \varepsilon_{7}}\right)
\end{aligned}
$$

where $i, j$ run through $1,2,3$ and 7 . Thus we have $\eta \operatorname{Sp}_{K_{0} / Q}(\xi)=$ $\xi^{2}$.

LEMMA 7. Suppose that $N\left(\varepsilon_{1}\right)=N\left(\varepsilon_{2}\right)=\cdots=N\left(\varepsilon_{7}\right)=-1$ and that $\sqrt{\Delta_{i j}} \notin \mathbf{Q}\left(\sqrt{d_{i}}, \sqrt{d_{j}}\right)$ for some $(i, j)$. Then we have $\bar{E}_{0}=$ $\left(E_{0}^{*}\right)^{+} E_{0}^{2}$.

Proof. Let $\eta \in \bar{E}_{0}$. By Lemma 1 we have

$$
\eta^{2}=\varepsilon_{1}^{x_{1}} \varepsilon_{2}^{x_{2}} \cdots \varepsilon_{7}^{x_{7}} \quad\left(x_{i} \in \mathbf{Z}\right) .
$$

Assume that every $x_{i}$ is odd. Taking the norm $N_{1}$ of (11), we have by Lemma 4 that $\varepsilon_{2}^{x_{2}} \varepsilon_{3}^{x_{3}} \varepsilon_{4}^{x_{4}}=1$ in $K_{1}$, because $K_{0}(\sqrt{\eta}) / K_{1}$ is a $(2,2)$-extension or $\sqrt{\eta}$ is contained in $K_{0}$. Therefore $\sqrt{\varepsilon_{2} \varepsilon_{3} \varepsilon_{4}} \in$ $K_{1}$, and then by (9) we have $\sqrt{\Delta_{23}} \in K_{1}=\mathbf{Q}\left(\sqrt{d_{2}}, \sqrt{d_{3}}\right)$. Similarly, taking the norms $N_{2}, N_{3}, N_{4}, N_{5}, N_{6}$ and $N_{7}$ of (11), we have $\sqrt{\Delta_{i j}} \in \mathbf{Q}\left(\sqrt{d_{i}}, \sqrt{d_{j}}\right)$ for every $(i, j)$. This contradicts the assumption. Hence there is an even integer among $x_{i}$ 's, and it follows from (2) of Lemma 3 that every $x_{i}$ is even. Therefore, $\eta \in\left(E_{0}^{*}\right)^{+} E_{0}^{2}$. Thus we have $\bar{E}_{0} \subseteq\left(E_{0}^{*}\right)^{+} E_{0}^{2}$.

The inverse inclusion $\left(E_{0}^{*}\right)^{+} E_{0}^{2} \subseteq \bar{E}_{0}$ is shown by the equations

$$
\sqrt{\eta} \sqrt{\operatorname{Sp}(\xi)}=\xi
$$


for $(\eta, \xi)=\left(\eta_{i j}, \xi_{i j}\right)$ and $\left(\eta_{i j k}, \xi_{i j k}\right)$, since $\left(E_{0}^{*}\right)^{+} E_{0}^{2} / E_{0}^{2}$ is represented by $\eta_{12}, \eta_{23}, \eta_{31}$ and $\eta_{123}$.

Proof of Theorem 1. First we assume that $\sqrt{\Delta_{i j}} \notin \mathbf{Q}\left(\sqrt{d_{i}}, \sqrt{d_{j}}\right)$ for some $(i, j)$.

Suppose that $Q_{K}=2$. Then there exists a unit $\eta \in \bar{E}_{0}$ such that $K_{0}(\sqrt{\eta})=K_{0}^{\prime}$ (Hasse [2, Satz 15]). By Lemma 7 we have $\eta=\varepsilon_{1}^{a_{1}} \varepsilon_{2}^{a_{2}} \cdots \varepsilon_{7}^{a_{7}} \varepsilon_{0}^{2}\left(a_{i} \in \mathbf{Z}, \varepsilon_{0} \in E_{0}\right)$ such that $\varepsilon_{1}^{a_{1}} \varepsilon_{2}^{a_{2}} \cdots \varepsilon_{7}^{a_{7}}$ is totally positive, and by (1) of Proposition $1 \eta=\eta_{12}^{b_{1}} \eta_{23}^{b_{2}} \eta_{31}^{b_{3}} \eta_{123}^{c} \varepsilon^{2}\left(b_{i}, c \in\right.$ $\left.\mathbf{Z}, \varepsilon \in E_{0}\right)$. Therefore it follows from (12) that

$$
K_{0}(\sqrt{\eta})=K_{0}\left(\sqrt{\Delta_{12}^{b_{1}} \Delta_{23}^{b_{2}} \Delta_{31}^{b_{3}} \Delta_{123}^{c}}\right) .
$$

Since $K_{0}^{\prime}=K_{0}(\sqrt{2})$ or $K_{0}\left(\sqrt{d_{0}}\right)$ according as $d_{0}=1$ or not, we have $K_{0}^{\prime}=K_{0}\left(\sqrt{A^{\prime}}\right)$ for some $A^{\prime}=A\left(e_{1}^{\prime}, e_{2}^{\prime}, e_{3}^{\prime}\right)$. Therefore

$$
K_{0}\left(\sqrt{\Delta_{12}^{b_{1}} \Delta_{23}^{b_{2}} \Delta_{31}^{b_{3}} \Delta_{123}^{c}}\right)=K_{0}\left(\sqrt{A^{\prime}}\right) .
$$

Thus we have

$$
\Delta_{12}^{b_{1}} \Delta_{23}^{b_{2}} \Delta_{31}^{b_{3}} \Delta_{123}^{c} \frac{\overline{2}}{2} A\left(e_{1}, e_{2}, e_{3}\right)
$$

for some $e_{i}=0,1$. Because, if $K_{0}(\sqrt{m})=K_{0}\left(\sqrt{A^{\prime}}\right)$ for a rational integer $m$ and $A^{\prime}=A\left(e_{1}^{\prime}, e_{2}^{\prime}, e_{3}^{\prime}\right)$, then $\mathbf{Q}\left(\sqrt{m / A^{\prime}}\right)$ is equal to $\mathbf{Q}$ or $\mathbf{Q}\left(\sqrt{m / A^{\prime}}\right)$ is a quadratic subfield of $K_{0}$, and so

$$
m=A^{\prime} d_{1}^{e_{1}^{\prime \prime}} d_{2}^{e_{2}^{\prime \prime}} d_{3}^{e_{3}^{\prime \prime}} r^{2}
$$

for some $e_{1}^{\prime \prime}, e_{2}^{\prime \prime}, e_{3}^{\prime \prime}=0,1$ and some $r \in \mathbf{Q}$. Therefore, putting $e_{i} \equiv e_{i}^{\prime}+e_{i}^{\prime \prime}(\bmod 2)(i=1,2,3)$, we have

$$
m=\frac{}{2} A\left(e_{1}, e_{2}, e_{3}\right)
$$

Conversely, if this equation (13) holds, then the square root of $\eta:=$ $\eta_{12}^{b_{1}} \eta_{23}^{b_{2}} \eta_{31}^{b_{3}} \eta_{123}^{c}$ generates $K_{0}^{\prime}$ over $K_{0}$, i.e., $K_{0}(\sqrt{\eta})=K_{0}^{\prime}$. Thus, by H. Hasse [2, Satz 15] we have $Q_{K}=2$.

Secondly, we assume that $\sqrt{\Delta_{i j}} \in \mathbf{Q}\left(\sqrt{d_{i}}, \sqrt{d_{j}}\right)$ for every $(i, j)$. Then it does not hold that

$$
\Delta_{12}^{b_{1}} \Delta_{23}^{b_{2}} \Delta_{31}^{b_{3}} \Delta_{123}^{c} \frac{\overline{2}}{2} A\left(e_{1}, e_{2}, e_{3}\right)
$$

for any $b_{i}, c, e_{i}=0,1$. 
In fact, by the assumption and by $\eta_{123}=\eta_{12} \eta_{36} \varepsilon_{6}^{-2}$ we have $K_{0}\left(\sqrt{\Delta_{i j}}\right)=K_{0}$ for every $(i, j)$ and $K_{0}\left(\sqrt{\Delta_{123}}\right)=K_{0}\left(\sqrt{\Delta_{12} \Delta_{36}}\right)=$ $K_{0}$. Consequently, we have

$$
\Delta_{12}^{b_{1}} \Delta_{23}^{b_{2}} \Delta_{31}^{b_{3}} \Delta_{123}^{c}=d_{1}^{\alpha_{1}} d_{2}^{\alpha_{2}} d_{3}^{\alpha_{3}} \neq A\left(e_{1}, e_{2}, e_{3}\right),
$$

where $\alpha_{i}=0$ or 1 .

In this case we can show that $Q_{K}=1$ as follows:

Assume that $Q_{K}=2$. Then there is a unit $\eta \in \bar{E}_{0}$ such that $K_{0}(\sqrt{\eta})=K_{0}^{\prime}$. By Lemma 1 we have $\eta^{2}=\varepsilon_{1}^{x_{1}} \varepsilon_{2}^{x_{2}} \cdots \varepsilon_{7}^{x_{7}}\left(x_{i} \in \mathbf{Z}\right)$. It follows from (2) of Lemma 3 that all $x_{i}$ are even or odd.

If all $x_{i}$ are even, then $\eta \in\left(E_{0}^{*}\right)^{+}$and we have $\eta=\eta_{12}^{b_{1}} \eta_{23}^{b_{2}} \eta_{31}^{b_{3}} \eta_{123}^{c} \varepsilon_{0}^{2}$ for some $b_{i}, c \in \mathbf{Z}$ and $\varepsilon_{0} \in E_{0}^{*}$. Since $\eta_{123}=\eta_{12} \eta_{36} \varepsilon_{6}^{-2}$, we obtain by the assumption that $\sqrt{\eta} \in K_{0}$, which contradicts that $K_{0}(\sqrt{\eta})$ is a quadratic extension over $K_{0}$. Therefore, all $x_{i}$ are odd. Then $\eta=$ $\sqrt{\varepsilon_{1} \varepsilon_{1} \cdots \varepsilon_{7}} \prod_{i=1}^{7} \varepsilon_{i}^{y_{i}}$ for some $y_{i} \in \mathbf{Z}$. Since $\varepsilon_{1} \varepsilon_{2} \ldots \varepsilon_{7}=\eta_{13} \eta_{23} \eta_{36} \varepsilon_{3}^{-2}$, we have

$$
\eta=\sqrt{\eta_{13}} \sqrt{\eta_{23}} \sqrt{\eta_{36}} \varepsilon_{3}^{-1} \prod_{i=1}^{7} \varepsilon_{i}^{y_{i}} .
$$

By (9) we have $\sqrt{\eta_{13}} r_{13} \sqrt{\Delta_{13}}=\xi_{13}$ for some $r_{13} \in \mathbf{N}$. And by the assumption we have $\Delta_{13}=d_{1}^{a_{1}} d_{3}^{a_{3}}$ for some $a_{1}, a_{3}=0,1$. Hence $\varepsilon_{1}^{a_{1}} \varepsilon_{3}^{a_{3}} \sqrt{\Delta_{13}}$ is totally positive. Moreover, from $\xi_{13}^{\sigma_{1}}<0, \xi_{13}^{\sigma_{2}}>0$, $\xi_{13}^{\sigma_{3}}<0$ it follows that $\varepsilon_{1} \varepsilon_{3} \xi_{13}$ is totally positive. Therefore

$$
\varepsilon_{1} \varepsilon_{3} \varepsilon_{1}^{a_{1}} \varepsilon_{3}^{a_{3}} \sqrt{\eta_{13}}=\frac{1}{r_{13}} \cdot \frac{\varepsilon_{1}^{a_{1}} \varepsilon_{3}^{a_{3}}}{\sqrt{\Delta_{13}}} \cdot \varepsilon_{1} \varepsilon_{3} \xi_{13}
$$

is totally positive, and then this unit is square in $K_{2}=\mathbf{Q}\left(\sqrt{d_{1}}, \sqrt{d_{3}}\right)$ (M. Hirabayashi and K. Yoshino [4, Proposition 2, IV]). So we can put

$$
\varepsilon_{1} \varepsilon_{3} \varepsilon_{1}^{a_{1}} \varepsilon_{3}^{a_{3}} \sqrt{\eta_{13}}=\varepsilon_{13}^{2}
$$

where $\varepsilon_{13}$ is a unit of $K_{2}$. In the same way we obtain

$$
\varepsilon_{2} \varepsilon_{3} \varepsilon_{2}^{b_{2}} \varepsilon_{3}^{b_{3}} \sqrt{\eta_{23}}=\varepsilon_{23}^{2}, \quad \varepsilon_{3} \varepsilon_{6} \varepsilon_{3}^{c_{3}} \varepsilon_{6}^{c_{6}} \sqrt{\eta_{36}}=\varepsilon_{36}^{2} \quad\left(b_{i}, c_{j}=0,1\right)
$$

where $\varepsilon_{23}$ and $\varepsilon_{36}$ are units of $K_{1}$ and $K_{6}$, respectively. Therefore we have

$$
\eta=\varepsilon_{13}^{2} \varepsilon_{23}^{2} \varepsilon_{36}^{2} \prod_{i=1}^{7} \varepsilon_{i}^{z_{i}} \quad\left(z_{i} \in \mathbf{Z}\right) .
$$


Since $\prod_{i=1}^{7} \varepsilon_{i}^{z_{i}}$ is totally positive, we have, as before,

$$
\prod_{i=1}^{7} \varepsilon_{i}^{z_{i}}=\eta_{12}^{\alpha_{1}} \eta_{23}^{\alpha_{2}} \eta_{31}^{\alpha_{3}}\left(\eta_{12} \eta_{36}\right)^{\alpha_{4}} \varepsilon_{0}^{2}
$$

for some $\alpha_{i} \in \mathbf{Z}$ and $\varepsilon_{0} \in E_{0}^{*}$. By the assumption each $\eta_{i j}$ is square in $\mathbf{Q}\left(\sqrt{d_{i}}, \sqrt{d_{j}}\right)$ and so is $\eta$ in $K_{0}$, which is also contradiction.

LEMma 8. If exactly one or two of $N\left(\varepsilon_{i}\right)(i=1,2, \ldots, 7)$ are +1 , then we have $\bar{E}_{0}=\left(E_{0}^{*}\right)^{+} E_{0}^{2}$.

Proof. It is enough to prove the following two Cases (1) and (2).

Case (1): $N\left(\varepsilon_{1}\right)=\cdots=N\left(\varepsilon_{5}\right)=-1$ and $N\left(\varepsilon_{6}\right)=N\left(\varepsilon_{7}\right)=+1$.

Let $\eta \in \bar{E}_{0}$ and let $\eta^{2}=\varepsilon_{1}^{x_{1}} \varepsilon_{2}^{x_{2}} \cdots \varepsilon_{7}^{x_{7}}\left(x_{i} \in \mathbf{Z}\right)$. By (2) of Lemma 3 we see that $x_{1}, x_{2}, \ldots, x_{5}$ are even. Then it follows from Lemma 4 that

$$
\begin{array}{ll}
\eta \eta^{\sigma_{4}}=\varepsilon_{1}^{x_{1}} \varepsilon_{4}^{x_{4}} \varepsilon_{7}^{x_{7}}=1 & \text { in } K_{4}, \\
\eta \eta^{\sigma_{5}}=\varepsilon_{2}^{x_{2}} \varepsilon_{5}^{x_{5}} \varepsilon_{7}^{x_{7}}=1 & \text { in } K_{5} .
\end{array}
$$

Now, we assume that $x_{7}$ is odd. Then $\varepsilon_{7}=1$ in $K_{4}=\mathbf{Q}\left(\sqrt{d_{1}}, \sqrt{d_{4}}\right)$ and in $K_{5}=\mathbf{Q}\left(\sqrt{d_{2}}, \sqrt{d_{5}}\right)$. Therefore, $\Delta_{7}=d_{1}^{e_{1}} d_{4}^{e_{4}}, \Delta_{7}=d_{2}^{e_{2}} d_{5}^{e_{5}}$ for some $e_{1}, e_{2}, e_{4}, e_{5}=0,1$. These equations lead that $\Delta_{7}=$ $\left(d_{1} d_{2} d_{3}\right)^{e_{1}}=d_{7}^{e_{1}}$, which is impossible (Kubota [5, Hilfssatz 9]). Thus $x_{7}$ is even. Similarly, by the equations

$$
\begin{array}{ll}
\eta \eta^{\sigma_{3}}=\varepsilon_{1}^{x_{1}} \varepsilon_{2}^{x_{2}} \varepsilon_{6}^{x_{6}}=1 & \text { in } K_{3}, \\
\eta \eta^{\sigma_{6}}=\varepsilon_{3}^{x_{3}} \varepsilon_{6}^{x_{6}} \varepsilon_{7}^{x_{7}}=1 & \text { in } K_{6},
\end{array}
$$

we see that $x_{6}$ is even. Therefore all $x_{i}$ are even and so $\eta \in E_{0}^{*}$. Thus $\bar{E}_{0} \subseteq\left(E_{0}^{*}\right)^{+} E_{0}^{2}$.

The inverse inclusion $\left(E_{0}^{*}\right)^{+} E_{0}^{2} \subseteq \bar{E}_{0}$ is shown by the equations (1) and (12).

Case (2): $N\left(\varepsilon_{1}\right)=N\left(\varepsilon_{2}\right)=\cdots=N\left(\varepsilon_{6}\right)=-1$ and $N\left(\varepsilon_{7}\right)=+1$.

Let $\eta \in \bar{E}_{0}$ and let $\eta^{2}=\varepsilon_{1}^{x_{1}} \varepsilon_{2}^{x_{2}} \cdots \varepsilon_{7}^{x_{7}}\left(x_{i} \in \mathbf{Z}\right)$. Then, by (2) of Lemma 3 we see that $x_{1}, x_{2}, \ldots, x_{6}$ are even. In the same way as in the proof of Case (1) we can show that $x_{7}$ is even and that $\bar{E}_{0}=\left(E_{0}^{*}\right)^{+} E_{0}^{2}$. 
Proof of Theorems 2 and 3. We only prove Theorem 2, because we prove Theorem 3 in a similar way.

Suppose that $Q_{K}=2$. Then there exists a unit $\eta \in \bar{E}_{0}$ such that $K_{0}(\sqrt{\eta})=K_{0}^{\prime}=K_{0}(\sqrt{A})$ where $A=A\left(e_{1}, e_{2}, e_{3}\right)$. By Lemma 8 and (2) of Proposition 1 we can put $\eta=\varepsilon_{7}^{a} \eta_{12}^{b_{1}} \eta_{23}^{b_{2}} \eta_{31}^{b_{3}} \varepsilon^{2}\left(a, b_{i} \in \mathbf{Z}, \varepsilon \in E_{0}\right)$ and we have

$$
K_{0}(\sqrt{\eta})=K_{0}\left(\sqrt{\Delta_{7}^{a} \Delta_{12}^{b_{1}} \Delta_{23}^{b_{2}} \Delta_{31}^{b_{3}}}\right) .
$$

Consequently,

$$
\Delta_{7}^{a} \Delta_{12}^{b_{1}} \Delta_{23}^{b_{2}} \Delta_{31}^{b_{3}}=A\left(e_{1}, e_{2}, e_{3}\right)
$$

Conversely, if this equation (14) holds, then a square root of $\eta:=$ $\varepsilon_{7}^{a} \eta_{12}^{b_{1}} \eta_{23}^{b_{2}} \eta_{31}^{b_{3}}$ generates $K_{0}^{\prime}$ over $K_{0}$, i.e., $K_{0}^{\prime}=K_{0}(\sqrt{\eta})$. Therefore we have $Q_{K}=2$.

Proof of Theorem 4.

Case (1): $N\left(\varepsilon_{1}\right)=N\left(\varepsilon_{2}\right)=N\left(\varepsilon_{3}\right)=N\left(\varepsilon_{4}\right)=-1$ and $N\left(\varepsilon_{5}\right)=$ $N\left(\varepsilon_{6}\right)=N\left(\varepsilon_{7}\right)=+1$.

Suppose that $Q_{K}=2$. Then there is a unit $\eta \in \bar{E}_{0}$ such that $K_{0}(\sqrt{\eta})=K_{0}^{\prime}$. By Lemma 1 and $\left(4_{1}\right)$ of Proposition 1 we have

$$
\eta^{2}=\eta_{23}^{x_{2}} \varepsilon_{5}^{x_{5}} \varepsilon_{6}^{x_{6}} \varepsilon_{7}^{x_{7}} \prod_{i=1}^{7} \varepsilon_{i}^{2 y_{i}}
$$

where $x_{i}, y_{i} \in \mathbf{Z}$. From (2) of Lemma 3 it follows that $x_{2} \equiv 0$ $(\bmod 2)$. Hence by Lemma 2 we see that $x_{5} \equiv x_{6} \equiv x_{7}(\bmod 2)$.

In the case that $x_{5} \equiv x_{6} \equiv x_{7} \equiv 0(\bmod 2)$, we have

$$
\eta=\varepsilon_{5}^{a_{5}} \varepsilon_{6}^{a_{6}} \varepsilon_{7}^{a_{7}} \eta_{23}^{b} \varepsilon_{0}^{2}
$$

for some $a_{i}, b=0,1$ and $\varepsilon_{0} \in E_{0}^{*}$. Therefore,

$$
K_{0}^{\prime}=K_{0}(\sqrt{\eta})=K_{0}\left(\sqrt{\Delta_{5}^{a_{5}} \Delta_{6}^{a_{6}} \Delta_{7}^{a_{7}} \Delta_{23}^{b}}\right)
$$

and then

$$
\Delta_{5}^{a_{5}} \Delta_{6}^{a_{6}} \Delta_{7}^{a_{7}} \Delta_{23}^{b}=A\left(e_{1}, e_{2}, e_{3}\right)
$$

for some $e_{i}=0,1$.

In the case that $x_{5} \equiv x_{6} \equiv x_{7} \equiv 1(\bmod 2)$, let

$$
\eta_{0}:=\sqrt{\varepsilon_{5} \varepsilon_{6} \varepsilon_{7}} \prod_{i=1}^{4} \varepsilon_{i}^{v_{\imath}} \quad\left(v_{i}=0 \text { or } 1\right)
$$


and let $\eta_{0}$ be totally positive. Then we have $\eta=\varepsilon_{5}^{a_{5}} \varepsilon_{6}^{a_{6}} \varepsilon_{7}^{a_{7}} \eta_{23}^{b} \eta_{0} \varepsilon_{0}^{2}$ where $a_{i}, b=0,1$ and $\varepsilon_{0} \in E_{0}^{*}$. Since $\varepsilon_{5}, \varepsilon_{6}, \varepsilon_{7}, \eta_{23}, \eta \in \bar{E}_{0}$, we see $\eta_{0} \in \bar{E}_{0}$. Then it follows from Lemma 5 that

$$
K_{0}\left(\sqrt{\eta_{0}}\right)=K_{0}\left(\sqrt{\xi^{*}\left(\eta_{0}\right)}\right)=K_{0}\left(\sqrt{\theta^{*}\left(\eta_{0}\right)}\right)=K_{0}\left(\sqrt{d^{*}\left(\eta_{0}\right)}\right)
$$

where $\xi^{*}\left(\eta_{0}\right), \theta^{*}\left(\eta_{0}\right)$ and $d^{*}\left(\eta_{0}\right)$ is defined by (2), (3) and (4), respectively. Here we take $s_{i}=0$ or $1(i=1,2,3)$ in accordance with

$$
\begin{aligned}
& \xi^{*}\left(\eta_{0}\right)=\left(\sqrt{\eta_{0}}+\sqrt{\eta_{0}} \sigma_{1}\right)^{2}, \quad \theta^{*}\left(\eta_{0}\right)=\left(\sqrt{\xi^{*}\left(\eta_{0}\right)}+\sqrt{\xi^{*}\left(\eta_{0}\right)} \sigma_{2}\right)^{2}, \\
& d^{*}\left(\eta_{0}\right)=\left(\sqrt{\theta^{*}\left(\eta_{0}\right)}+\sqrt{\theta^{*}\left(\eta_{0}\right)} \sigma_{3}\right)^{2},
\end{aligned}
$$

respectively. Therefore

$$
K_{0}^{\prime}=K_{0}(\sqrt{\eta})=K_{0}\left(\sqrt{\Delta_{5}^{a_{5}} \Delta_{6}^{a_{6}} \Delta_{7}^{a_{7}} \Delta_{23}^{b} d^{*}\left(\eta_{0}\right)}\right)
$$

and then we have

$$
\Delta_{5}^{a_{5}} \Delta_{6}^{a_{6}} \Delta_{7}^{a_{7}} \Delta_{23}^{b} d^{*}\left(\eta_{0}\right) \underset{2}{=} A\left(e_{1}, e_{2}, e_{3}\right)
$$

for some $e_{i}=0,1$.

Conversely, if the equation (15) or (16) holds, the square root of $\eta:=\varepsilon_{5}^{a_{5}} \varepsilon_{6}^{a_{6}} \varepsilon_{7}^{a_{7}} \eta_{23}^{b}$ or $\varepsilon_{5}^{a_{5}} \varepsilon_{6}^{a_{6}} \varepsilon_{7}^{a_{7}} \eta_{23}^{b} \eta_{0}$ generates $K_{0}^{\prime}$ over $K_{0}$, respectively, i.e., $K_{0}^{\prime}=K_{0}(\sqrt{\eta})$. Then we have $Q_{K}=2$.

Case (2): $N\left(\varepsilon_{1}\right)=N\left(\varepsilon_{2}\right)=N\left(\varepsilon_{3}\right)=N\left(\varepsilon_{7}\right)=-1$ and $N\left(\varepsilon_{4}\right)=$ $N\left(\varepsilon_{5}\right)=N\left(\varepsilon_{6}\right)=+1$.

Suppose that $Q_{K}=2$. Then by Lemma 1 and $\left(4_{2}\right)$ of Proposition 1 we have

$$
\eta^{2}=\varepsilon_{4}^{x_{4}} \varepsilon_{5}^{x_{5}} \varepsilon_{6}^{x_{6}} \eta_{123}^{z} \prod_{i=1}^{7} \varepsilon_{i}^{2 y_{i}}
$$

where $x_{i}, y_{i}, z \in \mathbf{Z}$. Then it follows from (2) of Lemma 3 that $z \equiv 0$ $(\bmod 2)$, and from Lemma 2 that $x_{4} \equiv x_{5} \equiv x_{6}(\bmod 2)$.

If $x_{4} \equiv x_{5} \equiv x_{6} \equiv 0(\bmod 2)$, then $\eta \in\left(E_{0}^{*}\right)^{+}$. By $\left(4_{2}\right)$ of Proposition 1 we have $\eta=\varepsilon_{4}^{a_{4}} \varepsilon_{5}^{a_{5}} \varepsilon_{6}^{a_{6}} \eta_{123}^{c} \varepsilon_{0}^{2}$ for some $a_{i}, c=0,1$ and $\varepsilon_{0} \in E_{0}^{*}$. Therefore,

$$
K_{0}(\sqrt{\eta})=K_{0}\left(\sqrt{\Delta_{4}^{a_{4}} \Delta_{5}^{b_{5}} \Delta_{6}^{b_{6}} \Delta_{123}^{c}}\right) .
$$

If $x_{4} \equiv x_{5} \equiv x_{6} \equiv 1(\bmod 2)$, taking norms $N_{1}$ and $N_{4}$ of the equation (17), we have by Lemma 4 that

$$
\begin{array}{ll}
\eta^{1+\sigma_{1}}=\varepsilon_{4}^{x_{4}} \varepsilon_{2}^{2 y_{2}} \varepsilon_{3}^{2 y_{3}} \varepsilon_{4}^{2 y_{4}}=1 & \text { in } K_{1}, \\
\eta^{1+\sigma_{4}}=\varepsilon_{4}^{x_{4}} \varepsilon_{1}^{2 y_{1}} \varepsilon_{7}^{2 y_{7}} \varepsilon_{4}^{2 y_{4}}=1 & \text { in } K_{4} .
\end{array}
$$


Then $\sqrt{\Delta_{4}}$ is contained in $K_{1} \cap K_{4}=\mathbf{Q}\left(\sqrt{d_{2} d_{3}}\right)$, and then $\Delta_{4}=1$ or $d_{2} d_{3}$, which is impossible (T. Kubota [5, Hilfssatz 9]).

Thus, if $Q_{K}=2$ we have the equation (18) and hence

$$
\Delta_{4}^{a_{4}} \Delta_{5}^{a_{5}} \Delta_{6}^{a_{6}} \Delta_{123}^{c}=A\left(e_{1}, e_{2}, e_{3}\right)
$$

for some $e_{i}=0,1$.

Conversely, when the equation (19) holds, we can show, as before, that $Q_{K}=2$.

Proof of Theorem 5. (1) Suppose that $N\left(\varepsilon_{1}\right)=N\left(\varepsilon_{2}\right)=N\left(\varepsilon_{3}\right)=-1$ and that $N\left(\varepsilon_{4}\right)=\cdots=N\left(\varepsilon_{7}\right)=+1$. By Lemma 1 and $\left(5_{1}\right)$ of Proposition 1 we have

$$
\eta^{2}=\varepsilon_{4}^{x_{4}} \varepsilon_{5}^{x_{5}} \varepsilon_{6}^{x_{6}} \varepsilon_{7}^{x_{7}} \prod_{i=1}^{7} \varepsilon_{i}^{2 y_{i}}
$$

for any $\eta \in \bar{E}_{0}$ where $x_{i}, y_{i} \in \mathbf{Z}$. Then by Lemma 2 we have the following three cases:

(i) $x_{4} \equiv x_{5} \equiv x_{6} \equiv x_{7} \equiv 0(\bmod 2)$;

(ii) Among $x_{4}, x_{5}, x_{6}$ and $x_{7}$, exactly one $x_{i}$ is even;

(iii) $x_{4} \equiv x_{5} \equiv x_{6} \equiv x_{7} \equiv 1(\bmod 2)$.

Case (i). We have $\eta \in\left(E_{0}^{*}\right)^{+}$and we may put $\eta=\varepsilon_{4}^{a_{4}} \varepsilon_{5}^{a_{5}} \varepsilon_{6}^{a_{6}} \varepsilon_{7}^{a_{7}}\left(a_{i} \in\right.$ $Z$ ). Then we obtain, as before,

$$
K_{0}(\sqrt{\eta})=K_{0}\left(\sqrt{\Delta_{4}^{a_{4}} \Delta_{5}^{a_{5}} \Delta_{6}^{a_{6}} \Delta_{7}^{a_{7}}}\right) .
$$

Case (ii). We first consider the case that $x_{4} \equiv x_{5} \equiv x_{6} \equiv 1, x_{7} \equiv 0$ (mod 2). Taking norms $N_{1}$ and $N_{4}$ of (20), we have

$$
\begin{array}{ll}
\eta^{1+\sigma_{1}}=\varepsilon_{4}^{x_{4}} \varepsilon_{2}^{2 y_{2}} \varepsilon_{3}^{2 y_{3}}=1 & \text { in } K_{1}=\mathbf{Q}\left(\sqrt{d_{2}}, \sqrt{d_{3}}\right), \\
\eta^{1+\sigma_{4}}=\varepsilon_{4}^{x_{4}} \varepsilon_{1}^{2 y_{1}} \varepsilon_{7}^{2 y_{7}}=1 & \text { in } K_{4}=\mathbf{Q}\left(\sqrt{d_{1}}, \sqrt{d_{4}}\right) .
\end{array}
$$

Then, as before, $\sqrt{\Delta_{4}}$ is contained in $\mathbf{Q}\left(\sqrt{d_{4}}\right)$, which is impossible.

Next we consider the other cases, for example, $x_{4} \equiv x_{5} \equiv x_{7} \equiv$ $1, x_{6} \equiv 0(\bmod 2)$. Let

$$
\eta_{0}:=\sqrt{\varepsilon_{4} \varepsilon_{5} \varepsilon_{7}} \prod_{i=1}^{3} \varepsilon_{i}^{v_{i}} \quad\left(v_{i}=0 \text { or } 1\right)
$$

and let $\eta_{0}$ be totally positive. Then we can prove the assertion in the same way as in the proof of Case (1) of Theorem 4. 
Case (iii). As before, taking norms $N_{1}, N_{2}, N_{3}$ and $N_{7}$ of (20), we obtain

$$
\begin{gathered}
\Delta_{4}=d_{2} \text { or } d_{3} ; \quad \Delta_{5}=d_{3} \text { or } d_{1} ; \quad \Delta_{6}=d_{1} \text { or } d_{2} ; \\
\Delta_{4} \Delta_{5} \Delta_{6}=d_{2} d_{3}, d_{3} d_{1} \text { or } d_{1} d_{2},
\end{gathered}
$$

which is impossible.

(2) Suppose that $N\left(\varepsilon_{1}\right)=N\left(\varepsilon_{2}\right)=N\left(\varepsilon_{6}\right)=-1$ and the others $N\left(\varepsilon_{i}\right)=+1$. We have by $\left(5_{2}\right)$ of Proposition 1

$$
\eta^{2}=\varepsilon_{3}^{x_{3}} \varepsilon_{4}^{x_{4}} \varepsilon_{5}^{x_{5}} \varepsilon_{7}^{x_{7}} \eta_{12}^{x_{1}} \prod_{i=1}^{7} \varepsilon_{i}^{2 y_{i}}
$$

for any $\eta \in \bar{E}_{0}$ where $x_{i}, y_{i} \in \mathbf{Z}$. By (2) of Lemma 3 we have $x_{1} \equiv 0$ $(\bmod 2)$. Therefore we obtain, as before, the following cases:

(i) $x_{3} \equiv x_{4} \equiv x_{5} \equiv x_{7} \equiv 0(\bmod 2)$;

(ii) Among $x_{3}, x_{4}, x_{5}$ and $x_{7}$, exactly one $x_{i}$ is even;

(iii) $x_{3} \equiv x_{4} \equiv x_{5} \equiv x_{7} \equiv 1(\bmod 2)$.

By the same argument in (1) of this proof we can prove the assertion for each case.

Proof of Theorem 6 . In the following we only consider the first case: $N\left(\varepsilon_{1}\right)=N\left(\varepsilon_{2}\right)=-1$, since the other cases are proved in the same way.

Let

$$
\eta_{0}:=\sqrt{\prod_{N\left(\varepsilon_{i}\right)=+1} \varepsilon_{i}^{u_{i}}} \cdot \prod_{N\left(\varepsilon_{i}\right)=-1} \varepsilon_{i}^{v_{i}} \quad\left(u_{i}, v_{i}=0 \text { or } 1\right)
$$

and let $\eta_{0}$ be totally positive.

For any $\eta \in \bar{E}_{0}$ we may put $\eta=\varepsilon_{3}^{a_{3}} \cdots \varepsilon_{7}^{a_{7}} \cdot \eta_{0}^{f}$ where $a_{i}, f=0$ or 1. Then we have, as before,

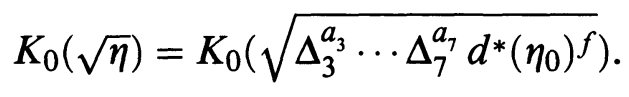

Thus we obtain that $Q_{K}=2$ if and only if

$$
\Delta_{3}^{a_{3}} \cdots \Delta_{7}^{a_{7}} d^{*}\left(\eta_{0}\right)^{f}=A\left(e_{1}, e_{2}, e_{3}\right),
$$

as desired. 


\section{REFERENCES}

[1] F. Halter-Koch, Arithmetische Theorie der Normalkörper von 2-Potenzgrad mit Diedergruppe, J. Number Theory, 3 (1971), 412-443.

[2] H. Hasse, Über die Klassenzahl abelscher Zahlkörper, Academie Verlag, Berlin, 1952 (reproduction: Springer Verlag, (1985)).

[3] M. Hirabayashi and K. Yoshino, Remarks on unit indices of imaginary abelian number fields II, Manuscripta Math., 64 (1989), 235-251.

[4] __ Unit indices of imaginary abelian number fields of type $(2,2,2), \mathrm{J}$. Number Theory, 34 (1990), 346-361.

[5] T. Kubota, Über den Bizyklischen Biquadratischen Zahlkörper, Nagoya Math. J., 10 (1956), 65-85.

[6] H. Wada, On the class number and the unit group of certain algebraic number fields, J. Fac. Sci. Univ. Tokyo, 13 (1966), 201-209.

Received July 30, 1991.

KanazaWa Institute of Technology

ISHIKAWA 921, JAPAN 


\section{PACIFIC JOURNAL OF MATHEMATICS}

Founded by

E. F. BeCKenbach (1906-1982) F. Wolf (1904-1989)

\section{EDITORS}

Sun-Yung A. Chang

(Managing Editor)

University of California

Los Angeles, CA 90024-1555

chang@math.ucla.edu

\section{F. Michael Christ}

University of California

Los Angeles, CA 90024-1555

christ@math.ucla.edu

\section{Herbert Clemens}

University of Utah

Salt Lake City, UT 84112

clemens@math.utah.edu

\author{
THOMAS ENRIGHT \\ University of California, San Diego \\ La Jolla, CA 92093 \\ tenright@ucsd.edu \\ Nicholas ERcolanI \\ University of Arizona \\ Tucson, AZ 85721 \\ ercolani@math.arizona.edu \\ R. FINN \\ Stanford University \\ Stanford, CA 94305 \\ finn@gauss.stanford.edu \\ VAUGHAN F. R. JONES \\ University of California \\ Berkeley, CA 94720 \\ vfr@math.berkeley.edu
}

Steven KerckhofF

Stanford University

Stanford, CA 94305

spk@gauss.stanford.edu

Martin SCHARLEMANN

University of California

Santa Barbara, CA 93106

mgscharl@math.ucsb.edu

Harold Stark

University of California, San Diego

La Jolla, CA 92093

V. S. VARADARAJAN

University of California

Los Angeles, CA 90024-1555

vsv@math.ucla.edu

\section{SUPPORTING INSTITUTIONS}

\author{
UNIVERSITY OF ARIZONA \\ UNIVERSITY OF BRITISH COLUMBIA \\ CALIFORNIA INSTITUTE OF TECHNOLOGY \\ UNIVERSITY OF CALIFORNIA \\ UNIVERSITY OF MONTANA \\ UNIVERSITY OF NEVADA, RENO \\ NEW MEXICO STATE UNIVERSITY \\ OREGON STATE UNIVERSITY
}

UNIVERSITY OF OREGON

UNIVERSITY OF SOUTHERN CALIFORNIA

STANFORD UNIVERSITY

UNIVERSITY OF HAWAII

UNIVERSITY OF UTAH

WASHINGTON STATE UNIVERSITY

UNIVERSITY OF WASHINGTON 


\section{PACIFIC JOURNAL OF MATHEMATICS}

Volume $164 \quad$ No. $1 \quad$ May 1994

Entropy versus orbit equivalence for minimal homeomorphisms

M. MiChaEL BoYle and DAVID E. HANDELMAN

The hyperspaces of infinite-dimensional compacta for covering and cohomological dimension are homeomorphic

TADEUSZ WLADYSLAW DOBROWOLSKI and LEONARD RUBIN

The index of transversally elliptic operators for locally free actions

JefFrey Stephen Fox and Peter Evarts Haskell

Unit indices of some imaginary composite quadratic fields

MiKiHITO HIRABAYASHI

Periodic points on nilmanifolds and solvmanifolds

EDWARD KEPPELMANN

Branched coverings of surfaces with ample cotangent bundle

MiCHAEL JEROME SPURR

Evolutionary existence proofs for the pendant drop and $n$-dimensional 147 catenary problems

ANDREW GUY STONE

Lattices of Lipschitz functions

NiKOLAI ISAAC WEAVER

Correction to: "Trace rings for verbally prime algebras" 